\title{
Efficiency and Environmental Metrics of Algal Fuel
}

\author{
Douglas Auld \\ Department of Economics and Finance, University of Guelph \\ Guelph, Ontario, Canada N1G 2W1 \\ Tel: 1-519-824-4120 E-mail: dauld@uoguelph.ca
}

Received: January 15, 2015 Accepted: February 9, 2015

doi:10.5296/emsd.v4i2.8182 URL: http://dx.doi.org/10.5296/emsd.v4i2.8182

\begin{abstract}
Energy efficiency, greenhouse gas (GHG) reduction effectiveness and cost are the major characteristics that determine the commercial production, consumption and potential subsidization of non-fossil, renewable fuels. This paper sets out a basic model by which the characteristics of algal fuel can be examined and in doing so, conclusions are reached regarding the likelihood of algal fuel becoming a major biofuel in the immediate and near-term future and the potential subsidy entitlement of the fuel.
\end{abstract}

Keyword: Biofuel, Algal fuel, Greenhouse gas

\section{Introduction}

For more than three decades, proponents of algal fuels have highlighted the apparent superior characteristics of these fuel compared to fossil fuels and first generation biofuels. ${ }^{1}$ Heading the list of advantages is the relatively small amount of land required to grow algae eschewing the debate over a trade-off between crops grown for fuel or food. In addition, algae can be grown in salt water, avoiding the practice of using fresh water which is required to produce corn and wheat ethanol. On the energy front, it is argued that algal fuel has a better EROI (energy return on investment) than biofuels such as cellulosic ethanol. (Liu, et al, 2013)

Furthermore, algae can be grown on land that is void of any vegetation, avoiding the deforestation that may take place when growing oilseed plants for biodiesel. Algae can be harvested daily or weekly compared to oilseed-based biofuels or corn and sugar ethanol. Finally, the fuels derived from algae are biodegradable and non-toxic. These are the claims. This paper provides a review of the key evidence related to energy, GHG emissions and cost

\footnotetext{
${ }^{1}$ For a summary of the early analysis of algal fuel, see Sheehan, (1998)
} 
associated with algal fuel, extracting some conclusions about the likelihood of algal fuel becoming a major biofuel in the immediate and near-term future and the potential subsidy entitlement of the fuel.

\section{The Quintessential Issues}

Energy efficiency, greenhouse gas (GHG) reduction effectiveness and cost are the major characteristics that determine the commercial production, consumption and potential subsidization of non-fossil, renewable fuels. There are two basic components to the energy efficiency issue: first, the ability of a given volume/weight of fuel to do work when combusted and second, the amount of energy necessary to produce a biofuel compared to the energy available from that biofuel. GHG effectiveness refers to the volume or weight of carbon equivalent emissions $\left(\mathrm{CO}_{2} \mathrm{ev}\right)$ for a given output of energy from one fuel compared to a baseline fuel. The cost issue revolves around (i) the price paid for each input times the units of input summed over all inputs required to manufacture a given fuel and (ii) the market price for the biofuel. A basic model will help to identify each characteristic and the interaction among these characteristics.

\section{Simple Model}

Let,

$\mathrm{J}^{0} \mathrm{X}_{\mathrm{i}}=$ megajoules (MJ) of energy released by the combustion of one liter of fuel $\mathrm{x}_{\mathrm{i}}$

$\mathrm{J}^{\mathrm{I}} \mathrm{x}_{\mathrm{k}}=$ megajoules $(\mathrm{MJ})$ of energy released when an input fuel source, $\mathrm{x}_{\mathrm{k}}$ is used to produce the output biofuel, $\mathrm{x}_{\mathrm{i}}$.

$\mathrm{Vx}$ = $=$ grams of $\mathrm{CO}_{2} \mathrm{ev}$ released into the atmosphere associated with the production and combustion of one liter of fuel $\mathrm{x}_{\mathrm{i}}$ including all by-products. ${ }^{2}$

$\mathrm{Px}^{\mathrm{I}}{ }_{\mathrm{i}}=\mathrm{cost}$ of all the factors of production (inputs) to manufacture one liter of fuel $\mathrm{x}_{\mathrm{i}}$

$\mathrm{Px}_{\mathrm{I}}^{0}=$ the market price for the fuel produced

When confronted with a biofuel that is technically capable of replacing a fossil fuel for a specific purpose, e.g. vehicle fuels, the following questions should be asked and answered: In relationship to the fossil fuel, is the fossil fuel replaced by the biofuel more/less energy efficient than the biofuel? Does the biofuel emit more, the same or less GHG emissions than the fossil fuel? Is the biofuel more, less or the same cost to produce as the fossil fuel on an energy equivalent basis?

For any given two fuels where $\mathrm{x}_{1}$ is a fossil fuel and $\mathrm{x}_{2}$ is a biofuel, if $\mathrm{Jx}_{1} \geq \mathrm{Jx}_{2}, \mathrm{Vx}_{1} \leq \mathrm{Vx} \mathrm{x}_{2}$ and $\mathrm{Px}^{\mathrm{I}}$ ${ }_{1} \leq \mathrm{Px}_{2}^{\mathrm{I}}$ there is no reason in the current period to replace the fossil fuel with the biofuel. ${ }^{3}$ In the case where $\mathrm{Jx}_{1}>\mathrm{Jx}_{2}, \mathrm{Vx}_{1}>\mathrm{Vx}_{2}$ and $\mathrm{Px}_{1}{ }_{1}=\mathrm{Px}_{2}{ }_{2}$, there exists the possibility of a trade-off between the characteristics of the two fuels in determining if the biofuel should replace the fossil fuel.

\footnotetext{
${ }^{2}$ The measure of carbon emissions, $\mathrm{CO}_{2} \mathrm{ev}$, comprises the five major greenhouse gasses weighted by their respective impact on global warming.

${ }^{3}$ It is assumed that the issue of the finite supply of fossil fuels is addressed by a rising marginal cost in the long run and resulting increase in price.
} 


\section{Macrothink}

The lower energy efficiency of $\mathrm{x}_{2},\left(\mathrm{Jx}_{1}>\mathrm{Jx}_{2}\right)$ may reduce the GHG effectiveness of $\mathrm{x}_{2}$ since more of that fuel must be consumed to replace the fossil fuel on an energy equivalent basis. It is therefore necessary to identify the 'energy adjusted GHG effectiveness' of the biofuel, $\mathrm{x}_{2}$. This 'adjusted effectiveness' we will denote as $\tilde{\mathrm{V}}$ which is equal to $\mathrm{Jx}_{1} / \mathrm{Jx}_{2}$. For example, it is well established that corn ethanol has $2 / 3$ energy per liter of fuel compared to gasoline such that $\tilde{\mathrm{V}} \mathrm{x}_{2}$ $=\mathrm{Vx}_{2} .\left(\mathrm{Jx}_{2} / \mathrm{Jx}_{1}\right)$ where $\mathrm{Jx}_{2} / \mathrm{Jx}_{1}=0.67$ and $\mathrm{x}_{1}$ and $\mathrm{x}_{2}$ are gasoline and corn ethanol respectively. (United States Department of Energy, 2014)

The second concept, the relationship between energy input and energy output is widely used in comparative fuel studies and is labelled the energy return on investment or EROI. Simply put, this is the ratio of the energy output of the fuel $\left(\mathrm{J}^{0} \mathrm{X}_{\mathrm{i}}\right)$ produced divided by the energy input needed to make the fuel $\left(\mathrm{J}^{\mathrm{I}} \mathrm{x}_{\mathrm{k}}\right)$. In general terms and to capture more than one energy input and energy output,

$J^{0} x_{i} / I^{I} x_{k}=\sum_{i=1}^{n} J_{i}^{0} / \sum_{k=1}^{m} J^{I} x_{k}$

An alternative measurement that focusses on a comparison between fossil and biofuels is the Energy Balance Ratio or EBR defined as

Total fossil energy input

Total Energy output from the biofuel

In most cases there will be only one final fuel. ${ }^{4}$ The energy return on investment is a measure of the technical EROI but to determine the economic return pertaining to energy, the formula must be modified to include input costs and output prices. This can be done in a straightforward manner my assigning the price (cost) of energy inputs and final price of the outputs such that

$\left(J^{\circ} x_{i} / I^{I} x_{k}\right)=\sum_{i=1}^{n} P x_{i} \cdot I^{0} x_{i} / \sum_{k=1}^{m} \quad P x_{k} \cdot J^{I} x_{k}$

Where $P x_{i}$ is the price of the $\mathrm{J}_{\text {th }}$ fuel output and $P x_{k}$ is the cost of the $\mathrm{k}_{\text {th }}$ input.

The following hypothetical example illustrates the connections between these characteristics.

Table 1. Energy output, cost and emissions factors for fuel comparison

\begin{tabular}{|c|c|c|c|}
\hline Fuel & Energy $/ \mathrm{kg}$ & Cost $/ \mathrm{kg}$ & Emissions (gms of CO2ev \\
\hline fossil & 120 joules & $\$ 1.50 / \mathrm{kg}$ & 75 \\
\hline biofuel & 60 joules & $\$ 1.00 / \mathrm{kg}$ & 25 \\
\hline
\end{tabular}

A quick glance at the data in the column, "Cost $/ \mathrm{kg}$ ", suggests that the biofuel is the least expensive but combined with the information in column 2 (indicating twice as much biofuel is

${ }^{4}$ One major exception is the output of anaerobic digestion of waste products where final outputs can be in the form of heat and electricity. 
needed to match the energy content of the fossil fuel), the energy efficiency cost of the biofuel is $\$ 2.00$ per kg. Disregarding the energy level, the biofuel has a major advantage over the fossil fuel, emitting only one- third of the GHG emissions. However, if twice as much biofuel has to be combusted to get the same energy per unit of fossil fuel, the advantage is reduced but not necessarily eliminated.

In the next three sections of the paper, we explore the energy efficiency, the GHG effectiveness and cost features of algal fuel particularly in relationship to the fossil fuel that might be replaced by the algal fuel. Some thoughts on the future of algal biofuel are included in the final section.

\section{Energy Efficiency Of Alga Fuel ${ }^{5}$}

As noted above, there are two basic issues around the question of the energy efficiency of any fuel: the energy or work the fuel does when combusted and the relationship between that energy output and the energy necessary to make the fuel itself. There is no simple answer to the question: what is the output of energy from a liter or gallon of algal fuel when combusted? Algal fuel can be produced from different strains of algae employing a variety of technical/manufacturing processes from a simple process to manufacture fuel for use in tractors to a highly sophisticated, capital intensive pilot facility designed to produce large quantities of fuel on an annual basis. ${ }^{6}$

While there is interest in several types of algal fuel, the majority of research and development is focussed on biodiesel which is the emphasis here. Regardless of the method employed to manufacture algae-based biodiesel fuel, there are four seminal phases involved: growing the algae, drying the algae, extracting the oil from the dried algae and fourth, using that oil in a process to produce a combustible fuel. There is a wide range of strains of algae but to be a feedstock for this biofuel, the criteria that must be considered are: the speed at which the algae grows without stimulants, the speed of growth in the presence of stimulants, the oil content of a given volume or weight of dried algae, the energy needed to remove the oil and how the oil responds to transesterification.(Campbell, 2008) Research published in 2011 demonstrated the sensitivity of the output of biofuel from algae to these factors. (Beal, 2011)

Restricting the analysis to biodiesel produced from algae, Shirvani found that algae based biodiesel required 2.5 times the energy of conventional biodiesel, almost as high energy fossil fuels. (Shirvani,2011) This finding is supported by Liu. (Liu, 2013) In contrast, an Australian study that examined both energy efficiency and GHG emissions of algal fuel concluded that algal fuel had a lower EBR for certain methods of producing algal fuel compared to the greater energy balance of canola biodiesel or fossil biodiesel fuels. (Sander, 2011)

\footnotetext{
5 Most of the literature focusses on biodiesel made from algae or algal biodiesel or algal fuel. Algae can be grown and harvested to produce bio-ethanol, crude oil and jet fuel as well. Seehttps://www.google.ca/?gfe_rd=cr\&ei=hVTdU8bMB8LB8geLkYFQ\&gws_rd=ssl\#q=wh at+fuels+can+be+made+from+algae. The focus in this paper is mainly algal biodiesel.

${ }^{6}$ For a description of these processes see, http://science.howstuffworks.com/environmental/green-science/algae-biodiesel2.htm
} 


\section{Macrothink}

Energy use and GHG are often inextricably linked. In their life cycle analysis (LCA) of algae biodiesel fuel, Sander and Murthy examine in detail the energy needs to convert algae into biodiesel fuel. The authors isolated the energy used in each of the 24 steps from harvesting the algae to combustion in a vehicle. The largest energy use (and hence a source of GHG) was drying the algae to render it practical in the subsequent stages to manufacture biodiesel fuel. The authors conclude, "...one major obstacle in algae technology ....the need to efficiently process the algae into its usable components." (p 704) It should be noted that algae is very efficient when it comes to land use. While the productivity of the feedstock for this fuel is extraordinarily high (95,000 liters of oil feedstock per hectare of land compared to 200 to 2000 $1 /$ hectare of land used to grow oil crops such as sunflower and canola for biodiesel), this feature cannot compensative for the inefficiencies associated with converting the oil to fuel.

\section{GHG Emissions and Algal Fuel}

Recent research applying life cycle analysis (LCA) to a pilot-scale algae biofuel production facility indicates that algae based biofuel exhibits lower GHG emissions than fossil fuel or first generation (oil seed) biodiesel. (Liu, et al, 2013) This result supports an earlier result where research simulated a functional 1,000MJ energy algae biodiesel production facility. (Sander, op. cit) These and other optimistic findings regarding the GHG advantage of algae biodiesel and not shared universally. In their life cycle analysis of algae- based biodiesel, other authors have concluded that the fuel has only the potential to be less carbon intensive than fossil fuel or first generation biodiesel:"Biodiesel from advanced biomass can realize its inherent environmental advantages of GHG emissions reduction once every step of the production chain is fully optimized and de-carbonized." (Shirvani, 2012, pages 94-5)) The model in this study examined both the energy efficiency issue and GHG emissions levels associated with data collected from the pilot scale facility. The results indicate that existing pilot-scale facilities have emission levels on par with conventional biofuels. While not encouraging initially, the research suggests there are significant opportunities to optimize the processes of production related to the selection of algae strain and energy recycling that would reduce GHG emissions. These projections about how technological improvements would impact the industry are inherently uncertain and should be interpreted guardedly.

An Australian study published in 2011 was designed to measure the GHG emissions as well as the economic viability associated with the production of algal fuel with different scenarios for carbon dioxide supplementation, algae production rates and incorporating the economic use of bi -products. (Campbell, 2011) For purposes of comparison, the GHG emissions were computed for the entire life cycle of a fuel, with and without recycling the residual waste to produce electricity by means of anaerobic digestion. The latter scenario resulted in net GHG emissions that were negative. Overall, algal biodiesel had emissions rates that were half those of canola-based biodiesel and one fifth of the GHG emissions from fossil diesel. The authors caution that the result is an optimistic estimate because there are no known pilot studies where algae was being produced at the rate assumed in this research. Algae contamination may also lower these estimates

An important GHG emissions consideration for the possible future use of algal fuel in vehicles 
is the U.S. Energy Independence and Security Act of 2007, administered by the Environmental Protection Agency (EPA) of the US government. The Act, among other things, establishes the life-cycle emissions eligibility requirements for renewable fuels. (United States, EPA, 2010) The threshold for biomass-based diesel or advanced biofuels such as algal fuel is 50\%: that is, on a life-cycle, energy equivalent basis, the fuel must emit $50 \%$ less GHG than fossil diesel fuel. The report concluded with a caveat that biodiesel from algal oils will comply with this threshold should they reach commercial production. The report notes that the conclusions reached by the EPA were based on an in depth review of the evidence from independent studies as well as the Agency's revised approach to lifecycle analysis for GHG emissions.

\section{The Stubborn Costs of Production}

Even if the estimates of EROI and GHG emissions reductions demonstrate a distinct advantage of algal fuel compared to first generation biofuels and fossil fuels, practically all studies to date have underscored the persistent high cost of producing algal fuel. To a significant degree, this cost is tied directing to the fundamental steps required to manufacture the fuel. To appreciate this somewhat binding constraint on the commercialization of algal fuel, a brief summary of these processes is found below. The purpose of this digest is to underscore the complexity of algal fuel production and highlight where technological advances are likely to be effective in reducing cost.

\subsection{Algae Harvesting and Recovery}

The major techniques currently employed in microalgae harvesting and recovery include centrifugation, flocculation, filtration and screening, gravity sedimentation, flotation, and electrophoresis techniques. Centrifugation is the use of centrifugal force to separate the solid material from the liquids in the harvested algae. Although centrifugation is an effective method to recover microalgae, the main disadvantage is the high investment in capital equipment and the operating costs needed to ensure the process is effective. If this process is to be used in large scale commercial operations to produce algae biodiesel, it will be important to locate facilities where the operating costs can be reduced. Detailed reviews of the procedure support this conclusion.(Grima, 2003)

Flocculation is a manufacturing activity where a solute particle in a solution forms a substance called a floc. Flocculation occurs when the particles strike each other. To speed up this process, chemicals called flocculants are often added. There are a variety of ways to approach flocculation to separate the solid and liquid material, all of which are costly in terms of energy inputs. An alternative approach is the technique of screening and filtering. Filtering allows the algal suspension is passed through a permeable membrane that retains the solids and allows the liquid to pass through. Similarly, screening involves passing the suspension through a screen. The particles that exceed the screen pore size are retained allowing the liquid to pass through the screen. Adding micro-strainers and vibrating screen filters fine-tunes the process of extracting solids from liquids but these refinements in the manufacturing process add to the cost of separating liquid from solid contributing to the total cost of the process.

The suspension could instead be subjected to filtration to separate liquid and solids. The liquid 
is forced through a filter by instigating a drop in pressure across the suspension. Gravity, vacuum, pressure, or centrifugal force can be used to create the pressure needed to complete the separation. The process can be carried out by surface filters which will result in the solids forming on the filter allowing the liquid to be drawn off. Filtration is not energy intensive but it is a slow process since regular backwashes of the material are needed as the microalgae bind within the material.

Gravity sedimentation separates a feed suspension into a concentrated slurry and clear liquid. Harvesting by sedimentation at natural gravity can be accomplished via lamella separators and sedimentation tanks. Finally, the procedure of flotation can be utilized to separate liquid and solid. Air or gas is injected into the suspension (of the liquids and solids) where the gaseous molecules adhere to the solid material leading to a separation of liquid and solid.

By way of a summary, numerous studies over the past decade or more have been initiated to examine the effectiveness and economic efficiency of recovering the feedstock needed for manufacturing algae-based diesel fuel. No single approach has been designated as a superior method. The choice of which harvesting technique to use depends on the species of microalgae and the final product desired. To date, producing algal fuel at a cost that would even be close to oil seed biodiesel or fossil diesel fuel has not been possible and until the cost of one or more these processes can be reduced, or the discovery of a new cost-effective process discovered, algae fuel production will be expensive. (Uduman, 2011; Chen, et al, 2011)

\section{Future Prospects for Algal Biodeisel}

There are a limited number of studies forecasting the commercial production of algal biodiesel. One of the more interesting models simulates the potential growth of algal biodiesel in competition with other biofuels. (Lee, 2011) The study uses, as a foundation, an extended version of the Taiwan computational general equilibrium model (CGE). With actual data for the period 2000 to 2009, the model is pushed out to the year 2040 incorporating assumption about improvements in energy savings in general, crude oil prices, inflation, population, tariffs and productivity. The strongest assumption in one scenario is a 25 per cent reduction in the production cost of commercial algal biodiesel each year which appears to be the consequence of a high level of government support for the production of algal biodiesel. The type and direction of the support is not specified. This implies that the low GHG emissions from algal biodiesel warrant government support. Without "strong support" by government and the substantial, annual reduction in algal biodiesel production costs, by 2040 this fuel plays no role in replacing other biofuels or fossil fuels.

A more optimistic outlook is provided by the research of Campbell, (op. cit.) In this three scenario analysis for the production of algal biodiesel in Australia, the authors report that if the production rate of algae is sufficiently high, algal biodiesel is competitive with canola-based biodiesel and fossil-fuel biodiesel. For their simulation, they examined the costs associated with a production rate of $15 \mathrm{~g} \mathrm{~m}^{-2} \mathrm{~d}$ and $30 \mathrm{~g} \mathrm{~m}^{-2} \mathrm{~d}$. The former has been achieved in practice; the latter only under laboratory conditions which suggests that it might be attainable. With that caveat, the analysis indicates that with the higher production level, algal biodiesel is competitive with other fuels. It should be noted that conditions for growing and harvesting 
algae are highly favorable in Australia.

\section{Conclusion}

Although the pace of research focussed on the conversion of algae to biofuels has accelerated in recent years, the excitement around this fuel is the result of positive results from small-scale pilot studies. The net energy balance is discouraging overall and while the land-yield (energy per hectare of land use) is higher than alternative biofuels, the production costs remain outside the realm of competing with first generation biofuels and fossil fuels. Based on the technical criteria for the commercial production and possible government support for algal fuel based on lower GHG emissions, further cost reductions must be achieved. In most cases, the research clearly identifies the superiority of algal fuels over first generation biofuels and fossil fuels.

The production and sale of algal biodiesel on a commercial scale remains an elusive goal although it is not for lack of trying. Data for the period up to 2010 indicates that close to a quarter of a billion dollars (US) has been spent by governments around the globe to promote and support research on algae-based biofuels. Figure 1 illustrates the overwhelming level of support in the US compared to other countries. In addition to government support, the private sector has invested hundreds of millions of dollars into research and development focussed on the commercialization of algal fuel. It would not be surprising if the total investment, public and private, in algal fuel research and development amount to one billion dollars for the year 2014. Finally, will the sharp reduction in the price of crude oil have an impact on fuel from algae? Cost comparisons are often expressed in terms of the energy equivalent volume of conventional diesel and algae-based diesel. As the price of crude oil and derivative products rises and that of algae-based fuel declines, the latter becomes more attractive. Should the price of crude oil remain low for a long period of time, the development of algae-based fuel becomes less attractive from a cost-of-energy perspective. However, the environmental benefits of algae-based fuel will continue to make this an attractive option in the longer run.

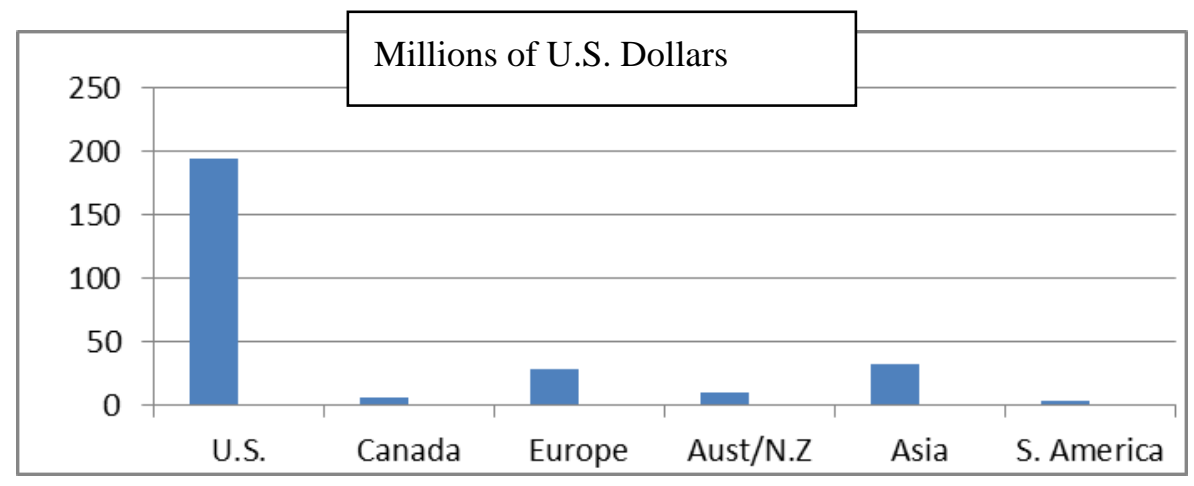

Figure 1. Government Financial Support for Algal Fuel [\$M U.S.], Cumulative to and including 2010 (Sikes, 2011)

\section{References}

Beal, Colin et al (2011), The Energy Return on Investment for Algal Biocrude: Results for a Research Production Facility, BioEnergy Researc. 
Campbell, Matthew, (2008). Biodiesel: Algae as a Renewable Source for Liquid Fuel." Guelph
Engineering
Journal,
$(1)$,
2-7.
ISSN:
1916-1107.

http://dx.doi.org/10.1016/j.biortech.2010.06.048

Campbell, Peter, Tom Beer and David Batten, (2011)," Life Cycle assessment of biodiesel production from microalgae in ponds",Bioresource Technology, 102(1), 50-56. DOI: 10.1016/j.biortech.2010.06.048

Chen, Chun-Yen, et al (2011), "Cultivation, photobioreacgtor design and harvesting of micro-algae for biodiesel production: A critical review," Bioresource Technology, 102(1), January 71-81.

Grima E., Molina, et al, (2003), "Recovery of microalgal biomass and metabolites: process options and economics", Biotechnol Adv, January 20(7-8), 491-515, http://openwetware.org/images/8/88/07-Algal_Food_Feed_Biofuels_final3.pdf

Lee, D.H. (2011). Algal biodiesel economy and competition among biofuels", Bioresource Technology, 102(1), January, 43-49. http://dx.doi.org/10.1007/s11367-010-0194-1

Liu, X., et al (2013),"Pilot-scale data provide enhanced estimates of the life cycle energy and emission profile of algae biofuels produced via hydrothermal liquefaction", Bioresource Technology, 148, November,163-7. http://dx.doi.org/10.1007/s11367-010-0194-1

Sander, Kyle and Ganti S. Murthy, (2010). Life cycle analysis of algae biodiesel" International Journal of Life cycle Assessment, 15, 704-14, DOI 10.1007/s11367-010-0194-1

Sheehan, John, et al (1998), A Look Back at the U.S. Department of Energy's Aquatic Species Program-Biodiesel from Algae, Office of Fuels Development, U.S. Department of Energy, Washington, DC

Shirvani, Tara, (2012). The environmental feasibility of algae biodiesel production, Applied Petrochemical Research, November, Volume 2, 93-95. http://dx.doi.org/10.1039/c1ee01791h

Shirvani, Tara, et al, (2011). Lifecycle energy and greenhouse gas analysis for algae-derived biodiesel. Energy and environmental Science, \# 10 DOI: $10.1039 /$ clee01791h

Sikes, Karen, Martijn Van Walwijk and Ralph McGill, (2011), Algae as a Feedstock for Biofuels, International Energy Agency, Advanced Motor Fuels

Uduman, Nyomi, et al (2010). Dewatering of microalgal cultures: A major bottleneck to algae-based fuels, Journal of Renewable and Sustainable Energy, 2, 012701. http://dx.doi.org/10.1063/1.3294480

United States Environmental Protection Agency, (2010), EPA Lifecycle Analysis of Greenhouse Gas Emissions from Renewable Fuels, Office of Transportation and Air Quality, Washington.

United States, (2014). Alternative Fuels Data Center-Fuel Properties Comparison, Energy Efficiency and Renewable Energy, Washington, D.C. 


\section{Macrothink \\ Environmental Management and Sustainable Development \\ ISSN 2164-7682}

\section{Copyright Disclaimer}

Copyright for this article is retained by the author(s), with first publication rights granted to the journal.

This is an open-access article distributed under the terms and conditions of the Creative Commons Attribution license (http://creativecommons.org/licenses/by/3.0/). 